UNIVERSIDADE DE SÃO PAULO

FACULDADE DE ODONTOLOGIA DE RIBEIRÃO PRETO

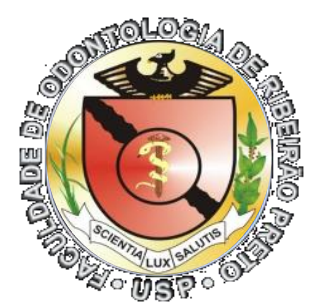

EFEITO DE DIFERENTES DENTIFRÍCIOS SOBRE O ESMALTE DENTAL: UM ESTUDO CLÍNICO DUPLO-CEGO CROSS-OVER

ANSELMO AGOSTINHO SIMIONATO

RIBEIRÃO PRETO 2018 
RESUMO 
EFEITO DE DIFERENTES DENTIFRÍCIOS SOBRE O ESMALTE DENTAL: UM ESTUDO CLÍNICO DUPLO-CEGO CROSS-OVER

Dissertação apresentada à Faculdade de Odontologia de Ribeirão Preto, da Universidade de São Paulo, para a obtenção do título de Mestre no programa de Reabilitação Oral.

Área de Concentração: Reabilitação Oral

Orientadora: Profa. Dra. Fernanda de Carvalho Panzeri Pires de Souza 
AUTORIZO A REPRODUÇÃO E DIVULGAÇÃO DO TEOR TOTAL OU PARCIAL DESTE TRABALHO POR QUALQUER MEIO CONVENCIONAL OU ELETRONICO, PARA FINS DE ESTUDO E PESQUISA, DESDE QUE CITADA A FONTE.

\section{FICHA CATALOGRÁFICA}

Simionato, Anselmo Agostinho

Efeito de diferentes dentifrícios sobre o esmalte dental: um estudo clínico duplo-cego cross-over.

116 p. :il. ; $30 \mathrm{~cm}$

Dissertação de Mestrado, apresentada à Faculdade de Odontologia de Ribeirão Preto/USP. Área de Concentração: Biologia Oral

Versão Original.

Orientadora: Pires-de-Souza, Fernanda de Carvalho Panzeri 
FOLHA DE APROVAÇÃO

\author{
ANSELMO AGOSTINHO SIMIONATO
}

\title{
EFEITO DE DIFERENTES DENTIFRÍCIOS SOBRE O ESMALTE DENTAL: UM ESTUDO CLÍNICO DUPLO-CEGO CROSS-OVER
}

Dissertação apresentada à Faculdade de Odontologia de Ribeirão Preto, da Universidade de São Paulo, para a obtenção do título de Mestre no programa de Reabilitação Oral.

Área de Concentração: Reabilitação Oral

Aprovado em:

\section{Banca Examinadora:}

1) Prof.(a) Dr. (a)

Instituição:

Julgamento: Assinatura:

2) Prof.(a) Dr. (a)

Instituição:

Julgamento: Assinatura:

3) Prof.(a) Dr. (a)

Instituição:

Julgamento: Assinatura: 
RESUM। 
RESUM। 
Aos meus pais, Agostinho e Roseli, pelo apoio em todas as escolhas, sucessos e fracassos que eu tive em minha vida, sempre com paciência, compreensão e muito amor. Pela luta e perseverança que tiveram em suas vidas e objetivos pessoais e para a construção de nossa família, nunca exigindo nada em troca, mas sempre com um sorriso no rosto a cada degrau que eu e meus irmãos conseguimos escalar. Por todos os valores que me foram passados, de honestidade, humildade e ética, que ditam os rumos atuais de minha vida e me norteiam neste caminho. Saibam que eu sou muito grato a tudo que vocês me proporcionaram e proporcionam até hoje, sem vocês eu não seria nada! Tudo o que faço é para vocês e por vocês, que me prepararam tanto para a vida.

Ao meu pai, Agostinho, que me traz tantas lembranças e me deixa tanta saudade, agradeço todo o incentivo para eu estudar e continuar estudando. Sempre lembro do senhor e do seu sorriso e a cada vez que a vida me põe cabisbaixo essa lembrança me faz erguer a cabeça e seguir em frente. Sentirei sua falta sempre e essa dor nunca desaparecerá, no entanto, saiba que você continua vivo dentro de mim e meu esforço é para ser um dia como o senhor foi.

À minha mãe, Roseli, por todo apoio ao longo de minhas conquistas e compreensão em todas as fases que tivemos, tanto ruins, quanto boas. Saiba que você é meu maior exemplo de bondade, de perseverança e exemplo também como educadora e professora. Você me inspira e me dá forças todos os dias para continuar!

Aos meus irmãos, Arnaldo e Ana Carolina, pela compreensão e paciência em todos as escolhas que eu fiz em minha vida, principalmente na minha carreira.

Aos meus avós, Vô Vino e Vó Zira, e, Vô Jacob e Vó Cidinha, por sempre me receberem de braços abertos. Saibam que os ensinamentos que promoveram em minha vida foram essenciais para a formação da pessoa que sou hoje! 
Aos meus avôs, Vô Jacob e Vô Vino, por serem grandes exemplos de trabalho, superação e persistência.

Aos meus tios, Ana, Ângela, Eugenio, Judite, Marlene, José, Rejane, Simone e Rivaldo, por todos os ensinamentos e por fazerem parte da minha vida.

À minha madrinha, Tia Judite, por ser a pessoa que mais acredita em mim no mundo todo. Por toda a compreensão, apoio e pelos puxões de orelha.

Ao meu tio, Rivaldo, pelo apoio em todos os momentos difíceis que passei nos últimos tempos. Pelo ouvido amigo e atento, e também pelos conselhos. Você é um dos exemplos de trabalho, força e dedicação que me inspiram nos dias de hoje.

Ao amigo, José Carlos Ferreira Júnior, por acompanhar toda a minha trajetória desde a graduação. Pelas palavras amigas, pelas trocas de ideias e conhecimentos. Por todo o apoio nos momentos difíceis e felicidade nos momentos bons. Sua amizade me proporcionou engrandecimento pessoal e amadurecimento. Saiba que sou muito grato por tudo!

À professora Fernanda, que me orientou no mestrado com muita paciência, dedicação e apoio. Você me apresentou um mundo novo, com novas perspectivas e anseios, além de modelar muitos aspectos do profissional que vou ser no futuro. Muito Obrigado!

Aos participantes da pesquisa, meu muitíssimo obrigado! Sem vocês o estudo não poderia acontecer! 
RESUN 
À Profa. Dra. Fernanda de Carvalho Panzeri Pires de Souza, por me acolher na forma de orientado em seu laboratório e grupo de pesquisa. Seu exemplo como profissional marca a trajetória do meu mestrado através de sua sabedoria e competência. Sua habilidade ao lidar com a minha pessoa me marcará por toda a vida, sendo um dos referenciais que possuirei ao ingressar, de fato, em uma carreira docente. Sua experiência foi fundamental para que eu me desenvolvesse e sofresse menos com os percalços da vida e da pesquisa. Muito obrigado por não desistir de me incentivar e por abrir a minha mente diante dos inúmeros desafios que permeiam o dia-a-dia.

À Rafaella Tonani Torrieri, por toda a assistência, coleguismo e companheirismo dentro da realização da pesquisa. Seu compromisso e ajuda, nas diversas dificuldades e tomadas de decisões, foram fundamentais, além dos diversos incentivos e palavras doces que nas horas de dificuldade me apoiaram durante toda esta jornada. Você terá sempre minha admiração e gratidão!

Ao Prof. Dr. Osvaldo Luiz Bezzon por me ajudar no ingresso na pós-graduação e por me passar um pouco de sua experiência.

\section{À Profa. Dra. Marilena Chinali Komesu e à Profa. Dra. Mônica Campos} Serra, pelas oportunidades oferecidas para minha formação como Cirurgião-Dentista.

À Universidade de São Paulo e à Faculdade de Odontologia de Ribeirão Preto, na pessoa de sua diretora, Profa. Dra. Léa Assed Bezerra da Silva, pelas oportunidades oferecidas durante o mestrado. Me orgulho de ter me graduado e de realizar esta importante etapa de minha formação acadêmica dentro desta casa.

Ao Programa de Pós-graduação em Reabilitação Oral, na pessoa do coordenador Prof. Dr. Ricardo Faria Ribeiro, pela oportunidade de cursar o Mestrado e pelo comprometimento com o programa.

À CAPES (Coordenação de Aperfeiçoamento de Pessoal de Nível Superior), pela bolsa concedida.

Aos Professores ligados às disciplinas cursadas durante o mestrado, pelos ensinamentos e conhecimentos compartilhados.

Ao Departamento de Materiais Dentários e Prótese, na pessoa de sua chefe, Profa. Dra. Valéria Oliveira Pagnano de Souza, pela autorização para a utilização dos 
laboratórios do departamento para a realização dos experimentos deste estudo. Aproveito também para agradecer as oportunidades oferecidas durante a realização das diversas monitorias e atendimentos em parceria com a Profa. Valéria, que me trouxeram engrandecimento profissional e aquisição de conhecimentos importantes para a prática clínica.

À Rafaella Tonani Torrieri, do Laboratório de Pesquisa "Prof. Dr. Heitor Panzeri"; à Ana Paula Macedo, do Laboratório de Pesquisa de Metrologia; e à Adriana Cláudia Lapria Faria Queiroz, do Laboratório de Estudos Biomecânicos em Prótese e Implantes. Agradeço pela dedicação, colaboração e contribuições durante a execução do projeto.

À Juliana Jendiroba Faraoni, pelo apoio durante a utilização do Microscópio Confocal de Varredura à Laser.

Às secretárias da pós-graduação Fernanda Talita de Freitas, Denise Martins Fontes Gonçalves e Mary Possani Carmessano, pela disponibilidade, paciência e colaboração em todo o processo do curso de mestrado em Reabilitação Oral.

À todos os Funcionários da Faculdade de Odontologia de Ribeirão Preto, da Universidade de São Paulo, em especial os funcionários do Departamento de Materiais Dentários e Prótese, por todo o carinho e companheirismo.

Ao colega, Sergio Augusto Vincentin pelo companheirismo, apoio e respeito dentro do ambiente de trabalho. Muito obrigado!

À colega, Gabriela Ayres de Souza, por todo o carinho, apoio e paciência durante o mestrado e a graduação.

À todos que, direta ou indiretamente, contribuíram para a realização deste trabalho. 
15 SUMO

Resuma 
SIMIONATO, A. A. Efeito de diferentes dentifrícios sobre o esmalte dental: um estudo clínico duplo-cego cross-over. Ribeirão Preto, 2019, 115p. Dissertação (Mestrado em Reabilitação Oral). Faculdade de Odontologia de Ribeirão Preto, Universidade de São Paulo.

\section{RESUMO}

O objetivo desta pesquisa foi avaliar, em um estudo in situ duplo cego e crossover, as alterações de cor, brilho, microdureza e rugosidade do esmalte dental submetido à escovação com dentifrícios clareadores e remineralizadores. Cento e vinte e dois fragmentos dentais bovinos $(6 \mathrm{~mm}$ x $6 \mathrm{mmx} 2 \mathrm{~mm})$ foram obtidos e esterilizados. Leituras iniciais (baseline) de cor ((Easyshade, VITA®, Bad Säckingen, Alemanha), rugosidade de superfície (Surftest SJ-201P Mitutoyo®, Kanagawa, Japão), brilho (Micro-Gloss $45^{\circ}$ BYK Gardner, Gerestried, Alemanha) e microdureza (Micro Hardness Tester HMV-2 Shimadzu®, Tóquio, Japão) foram obtidas. A partir da moldagem de 15 participantes selecionados para o estudo, de acordo com os critérios de inclusão determinados e aprovados no Comitê de Ética em Pesquisa, foram obtidos dispositivos bucais, sobre os quais quatro fragmentos de dentes bovinos foram incluídos ( 2 de cada lado, direito e esquerdo). Após a instalação, os participantes foram instruídos a utilizarem o dispositivo em período integral e realizarem a escovação por 7 dias, com escovas dentais macias recebidas (Orvital, Cotia, São Paulo, Brasil) e dentífrico convencional (Sorriso Dentes Brancos, Colgate-Palmolive) nas duas áreas do dispositivo (wash-out). Após esse período, os participantes receberam dois dos dentifrícios testados (Sorriso Dentes Brancos - Colgate-Palmolive; Colgate Luminous White - Colgate-Palmolive; Advance White - Arm \& Hammer; e Regenerate Enamel Science - Unilever) e foram orientados a utilizar, por 30 dias, um dentifrício de cada lado do dispositivo. Após esse período, receberam nova escova e os dois outros dentifrícios para utilização por período de 30 dias, conforme orientações prévias. Após o período de análise, os fragmentos foram desincluídos do dispositivo e submetidos às leituras finais de cor, brilho, microdureza e rugosidade. Dois fragmentos aleatórios foram selecionados após escovação com cada dentifrício testado para serem analisados em Microscopia Confocal de Varredura a Laser (Olympus Lext OLS4000, Olympus Europa Holding GmBH, Hamburg, Germany). Dois fragmentos não tratados foram considerados Controles. Os valores de cada propriedade foram analisados segundo ANOVA, medidas repetidas, Tukey $(\mathrm{p}<0,05)$ e os resultados não demonstraram diferenças estatisticamente significantes entre os grupos quando 
analisados cor, microdureza e brilho $(\mathrm{p}>0,05)$ e diferença significativa $(\mathrm{p}<0,05)$ para rugosidade de superfície entre os grupos CLW e RE. Concluiu-se que não há diferença na eficiência dos dentifrícios estudados quanto à cor, brilho e microdureza do esmalte dental. Dentifrícios com sílica hidratada produziram diferença na rugosidade de superfície do esmalte, porém não há relação entre esta propriedade e a abrasividade dos dentifrícios utilizados. 
RESUMC 
SIMIONATO, A. A. Effect of different toothpastes on enamel: a double-blind crossover clinical study. Ribeirão Preto, 2019, 115p. Dissertação (Mestrado em Reabilitação Oral). Faculdade de Odontologia de Ribeirão Preto, Universidade de São Paulo.

\begin{abstract}
The aim of this study was to evaluate the color stability, surface roughness, microhardness and gloss alterations of enamel submitted to brushing with over-thecounter toothpastes in a double-blind and cross-over study. One hundred and twenty-two bovine dental fragments $(6 \mathrm{~mm} \times 6 \mathrm{~mm} \times 2 \mathrm{~mm})$ were obtained and sterilized. Color baseline readings (Easyshade, VITA®, Bad Säckingen, Germany), surface roughness (Surftest SJ-201P Mitutoyo®, Kanagawa, Japan), gloss (Micro-Gloss $45^{\circ}$ BYK Gardner, Gerestried, Germany) and microhardness (Micro Hardness Tester HMV-2 Shimadzu®, Tokyo, Japan) were obtained. Fifteen participants selected for the study according to inclusion criteria, previous approved by the Research Ethics Committee, had the upper arch shaped. Devices were obtained from the upper arcade copy and installed. After the installation, the participants received a soft toothbrush (Orvital, Cotia, São Paulo, Brazil) and conventional toothpaste (Sorriso Dentes Brancos, Colgate-Palmolive). They were instructed to use the device in full time and to perform the brushing, according to the orientation: seven-day period, in the two areas (Wash-out period). After wash-out period, the participants received two of the toothpastes tested (White Tooth Smile - ColgatePalmolive; Colgate Luminous White - Colgate-Palmolive; Advance White - Arm \& Hammer; and Regenerate Enamel Science - Unilever) and were instructed to use a toothpaste on each side of the device for 30 days. After this period, they received a new toothbrush and the other two toothpastes to use for a period of 30 days, according to previous guidelines. After the analysis period, the fragments were removed from the device and submitted to final readings of color, gloss, microhardness and roughness. Two random fragments were selected after brushing with each toothpaste tested for analysis in Confocal Laser Scanning Microscopy (Olympus Lext OLS4000, Olympus Europe Holding GmBH, Hamburg, Germany). Two untreated fragments were considered Controls. The values of each property were analyzed by ANOVA, repeated measures, Tukey $(\mathrm{p}<0.05)$ and the results showed no statistical differences between groups when analyzed for color, microhardness and brightness $(\mathrm{p}>0.05)$ and significant difference $\mathrm{p}$ $<0.05$ ) for surface roughness between CLW and RE groups. It is concluded that there is
\end{abstract}


no difference in the efficiency of the toothpastes studied regarding the color, gloss and microhardness of enamel. Toothpastes with hydrated silica produced a difference in surface roughness of the enamel, however there is no relation between this property and the abrasiveness of the dentifrices used. 
23 3STRACT

Sumária 
ABSTRAC 24 


\section{SUMÁRIO}

RESUMO

ABSTRACT

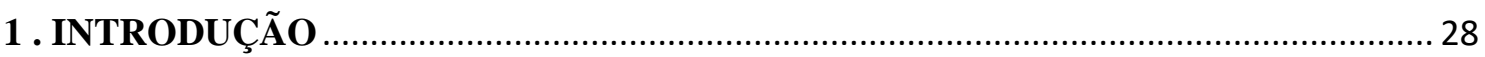

2 . PROPOSIÇÃ

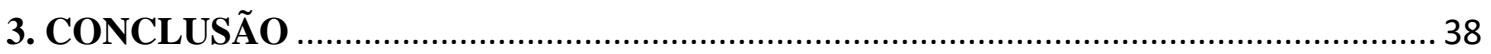

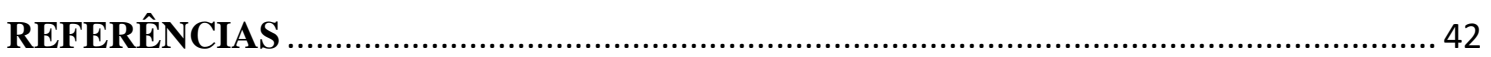

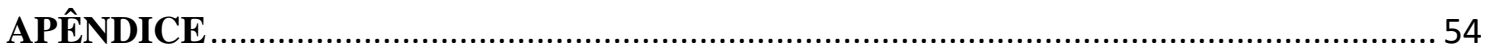

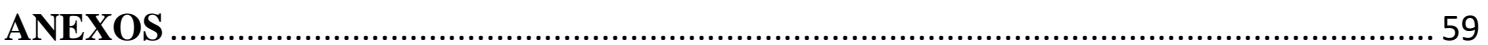


Intraducãa 



\section{1 . INTRODUÇÃO}

Um dos principais produtos de higiene utilizados são os dentifrícios, que são formulações utilizadas na higiene oral e que buscam oferecer limpeza dos dentes, com maior praticidade e menor desconforto durante a escovação dental. Em sua maioria foram desenvolvidos a partir de fórmulas existentes na medicina popular para impedir o progresso da doença cárie e para o alívio da dor de dente. Sua composição já incluiu plantas medicinais e ossos de animais $(1,2)$, devido a crenças populares nessas substâncias. Sua descrição data de 4000 anos antes de Cristo com ampla utilização, desde então, por motivos estéticos, para alivio de quadros dolorosos de etiologia dental e eliminação de odores desagradáveis provenientes da boca $(1,3)$.

A limpeza de todas as áreas dos dentes e a manutenção destas áreas limpas é uma necessidade para a saúde bucal. A falta de escovação e a alta frequência de consumo de alimentos cariogênicos podem ocasionar acúmulo de biofilme $(4,5)$, fator etiológico de algumas doenças, agudas ou crônicas, prejudiciais aos dentes e tecidos adjacentes (6), como cárie e periodontite $(7,8)$. Atualmente, essas doenças atingem parcela significativa da população, com doenças periodontais atingindo cerca de $47 \%$ da população adulta acima de 30 anos nos EUA e $78,1 \%$ no Brasil $(9,10)$ e a doença cárie atingindo ao menos um elemento dental de $90 \%$ da população brasileira adulta (10).

Além de diminuir a incidência de doenças, a higienização bucal também tem o objetivo de manter a estética dos dentes, pelo seu polimento e pela remoção de manchas extrínsecas causadas pela pigmentação da película adquirida (11-13) devido à ingestão de bebidas e alimentos, ou hábitos, como o fumo $(12,13)$.

Os dentifrícios são considerados cosméticos e são comercializados livremente sem necessidade de supervisão do dentista. $\mathrm{O}$ foco dos fabricantes de dentifrícios mudou conforme as necessidades de um mercado consumidor mais informado e exigente quanto aos benefícios dos produtos que utiliza.

A exigência de dentes cada vez mais brancos e a valorização da estética, por parte de grande parte da população, determinaram a inclusão de agentes terapêuticos e clareadores nas diversas marcas de dentifrícios que são encontrados nas prateleiras de farmácias e supermercados, e a cada dia ficam mais acessíveis à população de uma forma geral $(14,15)$. 


\section{INTRODUÇÃO}

A escovação associada à utilização de dentifrício clareador pode ser uma das opções para a minimização da alteração da cor dos dentes ao longo do tempo (16). Para que tal efeito seja obtido, dentífricos clareadores devem permitir a ação terapêutica dos seus agentes, sem que os tecidos bucais sejam lesionados, potencializando a limpeza e reduzindo o desgaste dental $(16,17)$.

A maioria dos dentifrícios clareadores possuem os mesmos ingredientes funcionais básicos que os dentifrícios convencionais e atuam removendo e prevenindo a formação de manchas extrínsecas (16). A inclusão de corantes, como o blue covarine, também é um meio de se obter melhores resultados de clareamento após a escovação (1821). Tais pigmentos alteram a reflexão de luz nos tecidos dentais, modificando a percepção de cor $(20,22-25)$.

Estudos na literatura relatam que esses produtos são eficientes $(26,27)$. Porém, outros estudos relatam que a alteração de cor de dentes após o uso de dentifrícios clareadores não é significativa ou não pode ser relacionada a condições clareadoras e sim abrasivas $(28,29)$. Superfícies mais lisas geralmente têm mais brilho (30-32). Dessa forma, o brilho de um dente está diretamente relacionado à lisura de superfície do esmalte dental (32). Abrasivos, surfactantes, polifosfatos e enzimas podem ser incluídos na formulação de dentifrícios clareadores aumentando a eficiência na remoção de manchas e dificultando a formação de nova película adquirida $(16,28)$.

A topografia superficial do esmalte dental tem influência direta na retenção de biofilmes, de forma que quanto maior a rugosidade, maior o acúmulo de biofilme $(3,33$, 34). Assim, a abrasividade de um dentifrício pode causar alterações de rugosidade da superfície dental, alterando o nível de retenção de biofilme sobre o dente.

Alguns dos principais problemas bucais que as pessoas enfrentam estão relacionados à perda de minerais no ambiente bucal devido a processos de cárie, lesões por erosão e abrasão ou hipersensibilidade dentária (35). As diversas formas de perda mineral dental em meio bucal mostram o interesse científico e comercial em produzir dentifrícios que são capazes de reverter ou estabilizar a perda mineral dos tecidos duros bucais (36).

Um dos agentes terapêuticos adicionados ao dentifrício é o flúor. Sua inclusão nas formulações teve grande destaque no cenário da Saúde Pública, pois promoveu a redução 
da prevalência de cárie tanto nas populações jovens quanto nas populações adultas, reduziu o número de casos onde o paciente sofria com quadros de dores intensas e com perdas dentais precoces (37). O efeito remineralizador do flúor é melhor visualizado nas populações mais jovens, onde estudos obtêm bons resultados com grupos experimentais nessa faixa etária (38).

Entretanto, muitas vezes, apenas a presença do flúor como agente terapêutico não é suficiente para reverter o processo de desmineralização. São necessárias outras ações para controlar esse processo, como o controle da ingestão de açúcares, da frequência e eficiência da remoção do biofilme. Assim, a adição de agentes remineralizadores aos dentifrícios, como o fosfato de cálcio e a arginina podem produzir efeitos sinérgicos na remineralização do esmalte $(39,40)$.

A utilização do peróxido de hidrogênio como agente clareador para dentes vitais é consagrada pelo seu uso em consultório (41), com a utilização de concentrações altas em ambiente controlado ou concentrações menores em ambiente doméstico associado ao acompanhamento do profissional responsável (42).

Apesar de efeitos adversos serem observados em alguns casos, a utilização do peróxido de hidrogênio como agente clareador tem suas indicações e mostra-se seguro em sua utilização (43). O peróxido de hidrogênio é capaz de reduzir a saturação da cor amarela dos dentes (44). Este componente também foi associado à redução na microdureza e alterações morfológicas do esmalte e da dentina após algumas condições experimentais, além de haver relatos de sensibilidade pós-operatória após clareamento com peróxido de hidrogênio $(45,46)$.

Dentifrícios contendo doses baixas de peróxido de hidrogênio, como o Colgate Luminous White Advanced, têm sido livremente comercializados a fim de reverterem os resultados ineficientes mencionados anteriormente. No entanto, a presença de agentes terapêuticos clareadores e abrasivos nos dentifrícios pode provocar alterações na superfície do esmalte, e seu uso contínuo pode provocar erosão dental $(15,47)$.

Apesar de encontrarmos resultados promissores quanto à remineralização do esmalte, não há dados na literatura sobre o efeito do uso desse tipo de dentifrício nas propriedades físicas e mecânicas dos dentes, bem como dados que comprovem a eficiência e segurança de dentifrícios com clareadores como agentes terapêuticos. 
Dessa forma, este estudo tem o objetivo de estudar o efeito de diferentes tipos de dentifrícios, incluindo àqueles de ação clareadora e remineralizadores, na alteração de cor, brilho, rugosidade de superfície e microdureza de esmalte dental. Para isso, propõese a realização de um estudo in situ, que garante fidelidade das condições do estudo às condições que os objetos de estudo são submetidos, e permite que a agressividade do ambiente oral, além dos efeitos da presença de saliva, atue diretamente sobre o experimento $(11,48)$. 
Propasicãa 



\section{2 . PROPOSIÇÃO}

Este estudo teve por objetivo avaliar in situ a alteração de cor, brilho, rugosidade de superfície, microdureza e topografia do esmalte dental submetido à escovação com diferentes dentifrícios.

A hipótese nula testada foi de que não haveriam alterações nas propriedades do esmalte dental, independentemente do tipo de dentifrício utilizado. 

Conclusãa 



\section{CONCLUSÃO}

Com base nos resultados encontrados no estudo, concluiu-se que:

- A escovação com os diferentes dentifrícios produziu alteração de cor no esmalte dental, independentemente do dentifrício utilizado, aumentando a luminosidade e diminuindo a saturação do croma amarelo.

- A escovação com os diferentes dentifrícios do estudo produziu aumento do brilho no esmalte dental, independentemente do dentifrício utilizado.

- Dentifrícios com sílica hidratada como abrasivo produziram maior alteração de rugosidade de superfície no esmalte dental, mas dentifrícios com o mesmo abrasivo produziram rugosidade de superfície diferentes.

- Não há relação entre a rugosidade de superfície do esmalte dental com a abrasividade dos dentifrícios utilizados no estudo.

- O flúor dos dentifrícios estudados colaboraram para aumentar a microdureza do esmalte dental após o uso de todos os dentifrícios estudados.

- Todos os dentifrícios estudados produziram superfícies de esmalte mais lisas após a escovação. 

Referências 



\section{REFERÊNCIAS}

1. Fischman SL. The history of oral hygiene products: how far have we come in 6000 years? Periodontology 2000. 1997; 15:7-14.

2. Lee SS, Zhang W, Li Y. The antimicrobial potential of 14 natural herbal dentifrices Results of an in vitro diffusion method study. Journal of the American Dental Association. 2004;135(8):1133-41.

3. Davies R, Scully C, Preston AJ. Dentifrices - an update. Medicina Oral Patologia Oral Y Cirugia Bucal. 2010;15(6):E976-E82.

4. Axelsson P, Lindhe J. THE SIGNIFICANCE OF MAINTENANCE CARE IN THE TREATMENT OF PERIODONTAL-DISEASE. Journal of Clinical Periodontology. 1981;8(4):281-94.

5. Paraskevas S, Timmerman MF, van der Velden U, van der Weijden GA. Additional effect of dentifrices on the instant efficacy of toothbrushing. Journal of Periodontology. 2006;77(9):1522-7.

6. Garcia-Godoy F, Hicks MJ. Maintaining the integrity of the enamel surface - The role of dental biofilm, saliva and preventive agents in enamel demineralization and remineralization. Journal of the American Dental Association. 2008;139:25S-34S.

7. Cochran DL. Inflammation and Bone Loss in Periodontal Disease. Journal of Periodontology. 2008;79(8s):1569-76.

8. Gomes VE, Silva DDd. A importância do controle de placa dental na clínica odontológica. Arquivos em Odontologia. 2010;46:22-7.

9. Eke PI, Dye BA, Wei L, Slade GD, Thornton-Evans GO, Borgnakke WS, et al. Update on Prevalence of Periodontitis in Adults in the United States: NHANES 2009 - 2012. Journal of periodontology. 2015;86(5):611-22.

10. Brasil MdS-. Saúde Bucal. Brasil: Governo Federal; 2008.

11. Camargo IMC, Saiki M, Vasconcellos MBA, Avila DM. Abrasiveness evaluation of silica and calcium carbonate used in the production of dentifrices. Journal of Cosmetic Science. 2001;52(3):163-7.

12. Watts A, Addy M. Tooth discolouration and staining: a review of the literature. British Dental Journal. 2001;190:309.

13. Kapadia Y, Jain V. Tooth Staining: A Review of Etiology and Treatment Modalities. Acta Scientific Dental Sciences [Internet]. 2018; 2(6):[67-70 pp.].

14. Demarco FF, Meireles SS, Masotti AS. Over-the-counter whitening agents: a concise review. Brazilian Oral Research. 2009;23(suppl 1):64-70. 


\section{REFERÊNCIAS}

15. Ozkan P, Kansu G, Ozak ST, Kurtulmus-Yilmaz S, Kansu P. Effect of bleaching agents and whitening dentifrices on the surface roughness of human teeth enamel. Acta Odontologica Scandinavica. 2013;71(3-4):488-97.

16. Joiner A. Whitening toothpastes: A review of the literature. Journal of Dentistry. 2010;38:E17-E24.

17. Hilgenberg SP, Souza Pinto SC, Farago PV, Santos FA, Wambier DS. Physical-chemical characteristics of whitening toothpaste and evaluation of its effects on enamel roughness. Brazilian Oral Research. 2011;25(4):288-94.

18. Ashcroft AT, Cox TF, Joiner A, Laucello M, Philpotts CJ, Spradbery PS, et al. Evaluation of a new silica whitening toothpaste containing blue covarine on the colour of anterior restoration materials in vitro. New technological breakthrough in whitening toothpastes. 2008;36:26-31.

19. Joiner A, Luo W. Tooth colour and whiteness: A review. Advances in tooth whitening: Perception, measurement and technology. 2017;67:S3-S10.

20. Kwon SR, Wertz PW. Review of the Mechanism of Tooth Whitening. Journal of Esthetic and Restorative Dentistry. 2015;27(5):240-57.

21. Tao D, Smith RN, Zhang Q, Sun JN, Philpotts CJ, Ricketts SR, et al. Tooth whitening evaluation of blue covarine containing toothpastes. Advances in tooth whitening: Perception, measurement and technology. 2017;67:S20-S4.

22. Harorlı OT, Barutcigil Ç. Color Recovery Effect of Commercial Mouth Rinses on a Discolored Composite. Journal of Esthetic and Restorative Dentistry. 2014;26(4):256-63.

23. Pan Q, Westland S. Tooth color and whitening - digital technologies. Digital Technologies in Oral \& Dental Research. 2018;74:S42-S6.

24. Pan Q, Westland S, Ellwood R. Evaluation of colorimetric indices for the assessment of tooth whiteness. 2018;76:132-6.

25. Joiner A. A silica toothpaste containing blue covarine: a new technological breakthrough in whitening. International Dental Journal. 2011;59(5):284-8.

26. Lima DANL, Silva ALFe, Aguiar FHB, Liporoni PCS, Munin E, Ambrosano GMB, et al. In vitro assessment of the effectiveness of whitening dentifrices for the removal of extrinsic tooth stains. Brazilian Oral Research. 2008;22:106-11.

27. Torres CRG, Perote L, Gutierrez NC, Pucci CR, Borges AB. Efficacy of Mouth Rinses and Toothpaste on Tooth Whitening. Operative Dentistry. 2012;38(1):57-62.

28. Alshara S, Lippert F, Eckert GJ, Hara AT. Effectiveness and mode of action of whitening dentifrices on enamel extrinsic stains. Clinical Oral Investigations. 2014;18(2):563-9.

29. Roselino Lde M, Chinelatti MA, Alandia-Roman CC, Pires-de-Souza Fde C. Effect of Brushing Time and Dentifrice Abrasiveness on Color Change and Surface Roughness of Resin Composites. Braz Dent J. 2015;26(5):507-13. 
30. Antonson SA, Yazici AR, Kilinc E, Antonson DE, Hardigan PC. Comparison of different finishing/polishing systems on surface roughness and gloss of resin composites. Journal of Color and Appearance in Dentistry. 2011;39:e9-e17.

31. Lefever D, Perakis N, Roig M, Krejci I, Ardu S. The effect of toothbrushing on surface gloss of resin composites. Am J Dent. 2012;25(1):54-8.

32. Chadwick AC, Kentridge RW. The perception of gloss: A review. Perception of Material Properties (Part I). 2015;109:221-35.

33. Nogueira RD, Silva CB, Lepri CP, Palma-Dibb RG, Geraldo-Martins VR. Evaluation of Surface Roughness and Bacterial Adhesion on Tooth Enamel Irradiated With High Intensity Lasers. Braz Dent J. 2017;28(1):24-9.

34. Marsh PD, Zaura E. Dental biofilm: ecological interactions in health and disease. J Clin Periodontol. 2017;44 Suppl 18:S12-S22.

35. Kelleher M, Bishop K. Tooth surface loss: an overview. Br Dent J. 1999;186(2):61-6.

36. Nunn JH. Prevalence of dental erosion and the implications for oral health. Eur J Oral Sci. 1996;104(2 ( Pt 2)):156-61.

37. Narvai PC, Frazao P, Roncalli AG, Antunes JL. [Dental caries in Brazil: decline, polarization, inequality and social exclusion]. Rev Panam Salud Publica. 2006;19(6):385-93.

38. Jensen ME, Kohout F. THE EFFECT OF A FLUORIDATED DENTIFRICE ON ROOT AND CORONAL CARIES IN AN OLDER ADULT-POPULATION. Journal of the American Dental Association. 1988;117(7):829-32.

39. Bou Chebel F, Zogheib CM, Baba NZ, Corbani KA. Clinical Comparative Evaluation of Nd:YAG Laser and a New Varnish Containing Casein Phosphopeptides-Amorphous Calcium Phosphate for the Treatment of Dentin Hypersensitivity: A Prospective Study. J Prosthodont. 2018.

40. Cantore R, Petrou I, Lavender S, Santarpia P, Liu Z, Gittins E, et al. In situ clinical effects of new dentifrices containing $1.5 \%$ arginine and fluoride on enamel de- and remineralization and plaque metabolism. 2013;24 Spec no A:A32-44.

41. França Moreira R, Pinheiro Santos F, Antero Santos E, Silva dos Santos R, José dos Anjos M, Sayão de Miranda M. Analysis of the Chemical Modification of Dental Enamel Submitted to 35\% Hydrogen Peroxide "In-Office" Whitening, with or without Calcium2017. 1-5 p.

42. Rezende M, Ferri L, Kossatz S, Loguercio AD, Reis A. Combined Bleaching Technique Using Low and High Hydrogen Peroxide In-Office Bleaching Gel. Operative Dentistry. 2016;41(4):388-96.

43. Goldberg M, Grootveld M, Lynch E. Undesirable and adverse effects of tooth-whitening products: a review. Clin Oral Investig. 2010;14(1):1-10. 


\section{REFERÊNCIAS}

44. Al-Omiri MK, Al Nazeh AA, Kielbassa AM, Lynch E. Randomized controlled clinical trial on bleaching sensitivity and whitening efficacy of hydrogen peroxide versus combinations of hydrogen peroxide and ozone. 2018;8(1):2407.

45. Tredwin CJ, Naik S, Lewis NJ, Scully C. Hydrogen peroxide tooth-whitening (bleaching) products: Review of adverse effects and safety issues. British Dental Journal. 2006;200(7):3716.

46. Joiner A. Review of the effects of peroxide on enamel and dentine properties. Journal of Dentistry. 2007;35(12):889-96.

47. Camargo SEA, Joias RP, Santana-Melo GF, Ferreira LT, El Achkar VNR, Rode SD. Conventional and whitening toothpastes: Cytotoxicity, genotoxicity and effect on the enamel surface. American Journal of Dentistry. 2014;27(6):307-11.

48. Briso AL, Fagundes TC, Gallinari MO, Moreira J, de Almeida L, Rahal V, et al. An In Situ Study of the Influence of Staining Beverages on Color Alteration of Bleached Teeth. Operative Dentistry. 2016;41(6):627-33.

49. Humphrey SP, Williamson RT. A review of saliva: Normal composition, flow, and function. Journal of Prosthetic Dentistry. 2001;85(2):162-9.

50. de Souza Fonseca Silva A, Ribeiro MC, Risso M. Biossegurança em odontologia e ambientes de saúde: Ícone; 2009.

51. Wiegand A, Kuhn M, Sener B, Roos M, Attin T. Abrasion of eroded dentin caused by toothpaste slurries of different abrasivity and toothbrushes of different filament diameter. 2009;37(6):480-4.

52. Pires-De-Souza FDP, Casemiro LA, Garcia LDR, Cruvinel DR. COLOR STABILITY OF DENTAL CERAMICS SUBMITTED TO ARTIFICIAL ACCELERATED AGING AFTER REPEATED FIRINGS. Journal of Prosthetic Dentistry. 2009;101(1):13-8.

53. Perez Mdel M, Ghinea R, Rivas MJ, Yebra A, Ionescu AM, Paravina RD, et al. Development of a customized whiteness index for dentistry based on CIELAB color space. Dent Mater. 2016;32(3):461-7.

54. Yu H, Jiang NW, Ye XY, Zheng HY, Attin T, Cheng H. In situ effect of Tooth Mousse containing CPP-ACP on human enamel subjected to in vivo acid attacks. J Dent. 2018;76:40-5.

55. Turssi CP, Messias DF, Corona SM, Serra MC. Viability of using enamel and dentin from bovine origin as a substitute for human counterparts in an intraoral erosion model. Braz Dent J. 2010;21(4):332-6.

56. Yassen GH, Platt JA, Hara AT. Bovine teeth as substitute for human teeth in dental research: a review of literature. J Oral Sci. 2011;53(3):273-82.

57. Zhang Z. Case-crossover design and its implementation in R. Ann Transl Med. 4. China2016. p. 341. 
58. Lombardi DA. The case-crossover study: a novel design in evaluating transient fatigue as a risk factor for road traffic accidents. Sleep. 2010;33(3):283-4.

59. Maclure M. The case-crossover design: a method for studying transient effects on the risk of acute events. Am J Epidemiol. 1991;133(2):144-53.

60. Jadad AR, Moore RA, Carroll D, Jenkinson C, Reynolds DJM, Gavaghan DJ, et al. Assessing the quality of reports of randomized clinical trials: Is blinding necessary? 1996;17(1):112.

61. Furberg CD, Soliman EZ. Double-blindness protects scientific validity. Journal of Thrombosis and Haemostasis. 2009;6(2):230-1.

62. Lacruz RS, Habelitz S, Wright JT, Paine ML. DENTAL ENAMEL FORMATION AND IMPLICATIONS FOR ORAL HEALTH AND DISEASE. Physiological reviews. 2017;97(3):939-93.

63. Roy S, Basu B. Mechanical and tribological characterization of human tooth. 2008;59(6):747-56.

64. Monterubbianesi R, Orsini G, Putignano A. New Trends of Colour and Background Effect in Restorative Dentistry2018.

65. Gomes MN, Francci C, Medeiros IS, Salgado NRDEGF, Riehl H, Marasca JM, et al. Effect of Light Irradiation on Tooth Whitening: Enamel Microhardness and Color Change. Journal of Esthetic and Restorative Dentistry. 2009;21(6):387-94.

66. Pecho OE, Ghinea R, Alessandretti R, Pérez MM, Della Bona A. Visual and instrumental shade matching using CIELAB and CIEDE2000 color difference formulas. 2016;32(1):82-92.

67. Johnston WM, Kao EC. Assessment of appearance match by visual observation and clinical colorimetry. J Dent Res. 1989;68(5):819-22.

68. Reis A, Loguercio AD. Materiais Dentários Diretos - Dos fundamentos à aplicação clínica. 2 ed: SANTOS EDITORA; 2007.

69. Li Y. Stain removal and whitening by baking soda dentifrice: A review of literature. Baking Soda Dentifrices and Oral Health. 2017;148(11, Supplement):S20-S6.

70. Brook AH, Smith RN, Lath DJ. The clinical measurement of tooth colour and stain. International Dental Journal. 2011;57(5):324-30.

71. Hara AT, Turssi CP. Baking soda as an abrasive in toothpastes: Mechanism of action and safety and effectiveness considerations. Baking Soda Dentifrices and Oral Health. 2017;148(11, Supplement):S27-S33.

72. St John S, White DJ. History of the Development of Abrasivity Limits for Dentifrices. The Journal of clinical dentistry. 2015;26(2):50-4.

73. Subramanian S, Appukuttan D, Tadepalli A, Gnana P, Samuel P, Victor DJ. The Role of Abrasives in Dentifrices. Journal of Pharmaceutical Sciences and Research. 2017;9(2):221-4. 


\section{REFERÊNCIAS}

74. Silva EMd, Maia JNdSMD, Mitraud CG, Russo JdES, Poskus LT, Guimarães JGA. Can whitening toothpastes maintain the optical stability of enamel over time? Journal of applied oral science : revista FOB. 2018;26:e20160460-e.

75. Williamson M. Toothpaste Abrasiveness Ranked by RDA (Relative Dentin Abrasion) Value https://www.williamsonperio.com/ [Available from: https://www.williamsonperio.com/. 76. de Moraes Rego Roselino L, Tirapelli C, de Carvalho Panzeri Pires-de-Souza F. Randomized clinical study of alterations in the color and surface roughness of dental enamel brushed with whitening toothpaste. J Esthet Restor Dent. 2018.

77. Kwon SR, R Kurti S, Oyoyo U, Li Y. Effect of various tooth whitening modalities on microhardness, surface roughness and surface morphology of the enamel2014.

78. Antón ARS, Lima MJP, Araújo RPCd. Hydrogen peroxide toothpaste: Whitening action? Rev odonto ciênc. 2009;24(2):161-7.

79. Lee Y-E, Park D-O, Jung Y-S, Song K-B. Evaluation of the whitening and remineralization effects of a mixture of amorphous calcium phosphate, hydroxyapatite and tetrasodium pyrophosphate on bovine enamel. 2016;40(2):92-9.

80. Yamamoto A, Rohumaa A, Hughes M, Vuorinen T, Rautkari L. Surface modification of birch veneer by peroxide bleaching. 2017;51(1):85-95.

81. Cvikl B, Lussi A, Moritz A, Flury S. Enamel Surface Changes After Exposure to Bleaching Gels Containing Carbamide Peroxide or Hydrogen Peroxide. Operative Dentistry. 2015;41(1):E39-E47.

82. Tao D, Sun JN, Wang X, Zhang Q, Naeeni MA, Philpotts CJ, et al. In vitro and clinical evaluation of optical tooth whitening toothpastes. Advances in tooth whitening: Perception, measurement and technology. 2017;67:S25-S8.

83. Whitehead SA, Shearer AC, Watts DC, Wilson NHF. Comparison of methods for measuring surface roughness of ceramic. Journal of Oral Rehabilitation. 1995;22(6):421-7.

84. Las Casas EB, Bastos FS, Godoy GCD, Buono VTL. Enamel wear and surface roughness characterization using 3D profilometry. 2008;41(12):1232-6.

85. Mullan F, Austin RS, Parkinson CR, Hasan A, Bartlett DW. Measurement of surface roughness changes of unpolished and polished enamel following erosion. PloS one. 2017;12(8):e0182406.

86. Anton R, Eva G, Grace R, Melissa A, Diah Ayu M. Potential Side Effects of Whitening Toothpaste on Enamel Roughness and Micro Hardness. 2015;11(4):239-42.

87. Pintado-Palomino K, Vasconcelos CV, Silva RJ, Fressatti AL, Motta BJ, Pires DESFC, et al. Effect of whitening dentifrices: a double-blind randomized controlled trial. Braz Oral Res. 2016;30(1):e82. 
88. Johannsen G, Tellefsen G, Johannsen A, Liljeborg A. The importance of measuring toothpaste abrasivity in both a quantitative and qualitative way. Acta Odontol Scand. 2013;71(34):508-17.

89. Hara AT, Turssi CP. Baking soda as an abrasive in toothpastes Mechanism of action and safety and effectiveness considerations. Journal of the American Dental Association. 2017;148(11):27S-33S.

90. Amaral CM, Rodrigues JA, Guilherme Erhardt MC, Barata Araujo MW, Marchi GM, Heymann HO, et al. Effect of Whitening Dentifrices on the Superficial Roughness of Esthetic Restorative Materials. Journal of Esthetic and Restorative Dentistry. 2006;18(2):102-8.

91. Sun Y, Li X, Deng Y, Sun JN, Tao D, Chen H, et al. Mode of action studies on the formation of enamel minerals from a novel toothpaste containing calcium silicate and sodium phosphate salts. Enamel erosion - Advanced mineralisation technology. 2014;42:S30-S8.

92. Vasconcelos AAMd, Cunha AGG, Borges BCD, Vitoriano JdO, Alves-Júnior C, Machado CT, et al. Enamel properties after tooth bleaching with hydrogen/carbamide peroxides in association with a CPP-ACP paste. Acta Odontologica Scandinavica. 2012;70(4):337-43.

93. Munoz CA, Stephens JA, Proskin HM, Ghassemi A. Clinical efficacy evaluation of calcium, phosphate, and sodium bicarbonate on surface-enamel smoothness and gloss. Compend Contin Educ Dent. 2004;25(9 Suppl 1):32-9.

94. Papas A, Russell D, Singh M, Kent R, Triol C, Winston A. Caries clinical trial of a remineralising toothpaste in radiation patients. Gerodontology. 2008;25(2):76-88.

95. Diamanti I, Koletsi-Kounari H, Mamai-Homata E, Vougiouklakis G. Effect of fluoride and of calcium sodium phosphosilicate toothpastes on pre-softened dentin demineralization and remineralization in vitro. Journal of Dentistry. 2010;38(8):671-7.

96. Rao A, Malhotra N. The role of remineralizing agents in dentistry: a review. Compend Contin Educ Dent. 2011;32(6):26-33; quiz 4, 6.

97. Gjorgievska ES, Nicholson JW. A preliminary study of enamel remineralization by dentifrices based on Recalden (CPP-ACP) and Novamin (calcium-sodium-phosphosilicate). Acta Odontol Latinoam. 2010;23(3):234-9.

98. Ganss C, Marten J, Hara AT, Schlueter N. Toothpastes and enamel erosion/abrasion Impact of active ingredients and the particulate fraction. 2016;54:62-7.

99. Jurema AL, Claudino ES, Torres CR, Bresciani E, Caneppele TM. Effect of Over-thecounter Whitening Products associated or Not with 10\% Carbamide Peroxide on Color Change and Microhardness: in vitro Study. J Contemp Dent Pract. 2018;19(4):359-66.

100. Assuncao CM, Lussi A, Almeida Rodrigues J, Carvalho TS. Efficacy of toothpastes in the prevention of erosive tooth wear in permanent and deciduous teeth. Clin Oral Investig. 2018. 


\section{REFERÊNCIAS}

101. Shen P, Bagheri R, Walker GD, Yuan Y, Stanton DP, Reynolds C, et al. Effect of calcium phosphate addition to fluoride containing dental varnishes on enamel demineralization. Australian Dental Journal. 2015;61(3):357-65.

102. Liu S, Wang H, Cheng Z, Liu H. Hexametaphosphate-capped quantum dots as fluorescent probes for detection of calcium ion and fluoride. 2016;232:306-12.

103. Zhu JJ, Tang ATH, Matinlinna JP, Hägg U. Acid etching of human enamel in clinical applications: A systematic review. Journal of Prosthetic Dentistry. 2014;112(2):122-35.

104. Da Cunha FB, Rodrigues ESBH, Freitas De Paula BL, Alencar CM, de Albuquerque Jasse FF, Silva CM. Effect of high concentrated fluoride-based dentifrice on the hardness, roughness, and color of the bleached enamel. J Conserv Dent. 2018;21(4):433-7.

105. Rakhmatullina E, Beyeler B, Lussi A. Inhibition of enamel erosion by stannous and fluoride containing rinsing solutions. Schweiz Monatsschr Zahnmed. 2013;123(3):192-8.

106. Oliveira MRC, Oliveira PHC, Oliveira LHC, Horliana ACRT, CÉSar PF, Moura SK, et al. Microhardness of bovine enamel after different fluoride application protocols. 2018; advpub.

107. ten Cate JM, Exterkate RAM, Buijs MJ. The Relative Efficacy of Fluoride Toothpastes Assessed with pH Cycling. 2006;40(2):136-41.

108. Joiner A. The bleaching of teeth: A review of the literature. 2006;34(7):412-9.

109. Neves JG, Danelon M, Pessan JP, Figueiredo LR, Camargo ER, Delbem ACB. Surface free energy of enamel treated with sodium hexametaphosphate, calcium and phosphate. 2018;90:108-12.

110. Jones SB, Davies M, Chapman N, Willson R, Hornby K, Joiner A, et al. Introduction of an interproximal mineralisation model to measure remineralisation caused by novel formulations containing calcium silicate, sodium phosphate salts and fluoride. J Dent. 2014;42 Suppl 1:S4652 .

111. Hornby K, Ricketts SR, Philpotts CJ, Joiner A, Schemehorn B, Willson R. Enhanced enamel benefits from a novel toothpaste and dual phase gel containing calcium silicate and sodium phosphate salts. Journal of Dentistry. 2014;42:S39-S45.

112. Parker AS, Patel AN, Al Botros R, Snowden ME, McKelvey K, Unwin PR, et al. Measurement of the efficacy of calcium silicate for the protection and repair of dental enamel. Journal of dentistry. 2014;42 Suppl 1:S21-9.

113. Dionysopoulos D, koliniotou-koumpia E, Tolidis K, Gerasimou P. Effect of Fluoride Treatments on Bleached Enamel Microhardness and Surface Morphology2017. 169-75 p.

114. Creeth JE, Karwal R, Hara AT, Zero DT. A Randomized in situ Clinical Study of Fluoride Dentifrices on Enamel Remineralization and Resistance to Demineralization: Effects of Zinc. 2018;52(1-2):129-38. 
115. Azevedo AM, Panzeri H, Prado CJ, De-Mello JD, Soares CJ, Fernandes-Neto AJ. Assessment in vitro of brushing on dental surface roughness alteration by laser interferometry. Braz Oral Res. 2008;22(1):11-7.

116. Robinson C, Connell S, Kirkham J, Brookes SJ, Shore RC, Smith AM. The Effect of Fluoride on the Developing Tooth. 2004;38(3):268-76. 

Apêndice 



\section{APÊNDICE}

\section{Documento de pesquisa 1 - TCLE do projeto assinado pelos pesquisadores responsáveis.}

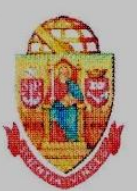

UNIVERSIDADE DE SÃO PAUIO

Faculdade de Odontologia de Ribeirão Preto

Departamento de Materiais Dentários e Protese

Programa de Pós-Graduaçäo em Reabilitaçäo Oral

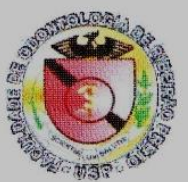

TERMO DE CONSENTIMENTO LIVRE E ESCLARECIDO

Nós, Profa. Dra. Fernanda de Carvahno Panzeri Pires-de-Souza (Responsável pelo Projeto) e Anselmo Agostinho Simionato (aluno de mestrado) convidamos vocë a participar do projeto intitulado "Efeito de diferentes dentifricios sobre o esmalte dental: um estudo in situ".

Esclarecemos que nosso objetivo é avaliar diferentes pastas de dente e sua ação sobre a superficie do dente. Para isso será realizado um questionario para identificar se você atende os criterios de inclusão na pesquisa.

Dessa maneira, se você aceitar participar do estudo é importante que saiba que:

- Serão realizadas óuas moldagens (cópia da parte superior da sua boca) e a partir delas seräo confeccionados modelos (reproduçăo dos tecidos bucais em gesso). Existe o risco de ânsia durante a moldagem, os procedimentos serão realizados pensando sempre em causar o menor desconforto possivel.

- Um dispositivo será obtido a partir do modelo. Este dispositivo receberá em sua superficie 4 fragmentos esterilizados de dente bovino $(6 \mathrm{~mm} \times 6 \mathrm{~mm} \times 2 \mathrm{~mm})$, que deverăo ser escovados da seguinte maneira:

\begin{tabular}{ll}
\multicolumn{1}{c}{ Pasta de Dente } & \multicolumn{1}{c}{$\begin{array}{c}\text { Periodo de Escovação } \\
\text { Wash-Out (10 Dispositivo) }\end{array}$} \\
1 & 7 dias (dois lados) \\
2 & 30 dias (lado direito) \\
Wash-Out (2 ${ }^{\circ}$ Dispositivo) & 30 dias (lado esquerdo) \\
3 & 7 dias (dois lados) \\
4 & 30 dias (lado direito) \\
Wash-Out: escovaçäo com apenas uma & 30 dias (lado esquerdo) \\
pasta de dente para padronizar o estudo.
\end{tabular}

- Havera quatro tioos de pastas de dente. O senhor (a) receberá duas pastas de cada vez em embalagens não identificadas, conforme distribuicão realizada pelos pesquisadores.

- Todos os materiais e procedimentos relacionados ao estudo seräo fornecidos pelos pesquisadores (instruçóes de escovação, instruções de uso das escovas de dente e pastas de dente)

- Serăo necessárias até 06 consultas, com intervalo minimo de 07 dias e máximo de 30 dias entre cada uma. 0 periodo total do estudo será de 80 dias. 0 agendamento será realizado sempre com 07 dias de antecedência.

- Os gastos decorrentes da participaçâo do estudo, tais como transporte e alimentação nos dias em que for necessária a presenca do senhor (a) para consultas ou exames seräo ressarcidos pelos pesquisadores deste estudo.

- Como beneficio prestado pelo estudo, o senhor (a) receberá um exame clinico detalhado da boca limpeza da superficie dental e instruçôes de higiene oral, ao término da participação no estudo.

- O estudo apresenta riscos como descolamento do fragmento dental bovino da superficie do dispositivo (com possivel risco de deglutiçâa) e lesōes em tecidos moles orais decorrentes da inserção incorreta do dispositivo na boca. A correta utilização do dispositivo conforme as orientacoes dos pesquisadores previne que aconteça maiores problemas Qualquer desconforto ou problema. causado pelo estudo, será de responsabilidade dos pesquisadores e consequentemente, será totalmente resolvido ou ressarcido pelos mesmos.

- Durante todo o periodo do estudo o senhor (a) pode solicitar maiores informações ou decidir retirar seu consentimento em participar sem que isto acarrete qualquer penalidade e näo cause prejuizo ao senhor (a). Todos os dados e materiais coletados serão mantidos em sigilo.

- Durante todo o periodo do estudo o senhor (a) será acompanhado pelos pesquisadores Prof Dra . Fernanda de Carvalho Panzeri Pires-de-Souza e Mestrando Anseimo Agostinho Smionato. a quem poderà contatar para conversar sobre assuntos 
55

APÊNDICE

relacionados ao tratamento, respectivamente nos números (16) 3315-3973 e (16) 3315 4081.

Para qualquer dúvida ou reclamação você poderá entrar em contato com a secretaria do Comitê de Etica em Pesquisa (CEP) pelo e-mail cep@forp.usp.br ou pelo telefone (16) 33150493 das $13: 30$ às 17:30. Sqy nome será mantido em anonimato.

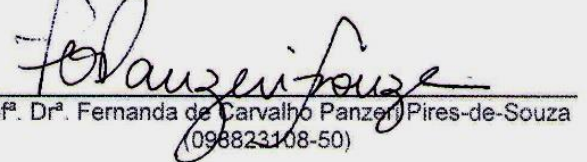

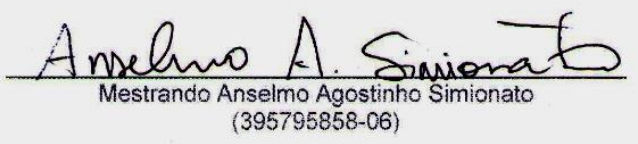

Compreendi todos os passos da pesquisa, concordo em participar da mesma e por isso assino este termo em duas vias, sendo uma de minha posse e a outra de posse dos pesquisadores:

Ribeirão Preto de de $201 \ldots$

Nome:

CPF: 
APÊNDICE 
ICE

Anexa 


\section{ANEXOS}

Anexo I: Parecer circunstanciado do CEP

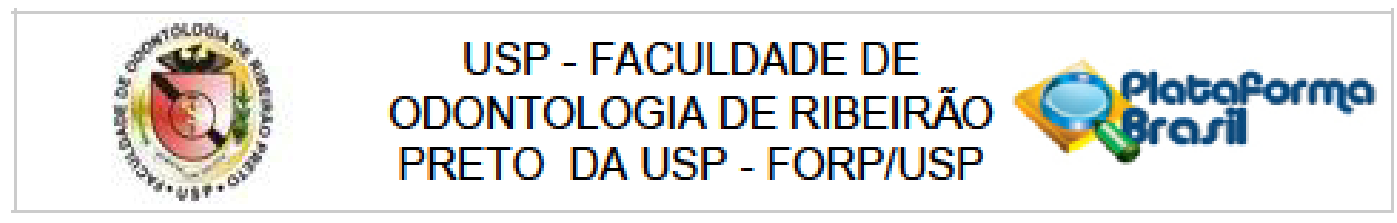

Contlnuş̧\$o do Parecer: 2.401.193

Objetivo Primário:

Este estudo tem por objetivo avaliar in situ a alteração de cor, brilho, rugosidade de superficie, micro dureza e topografia do esmalte dental

submetido à escovação com diferentes dentifricios.

\section{Avaliação dos Riscos e Beneficios:}

Riscos:

Descolamento do fragmento dental bovino da superficie do dispositivo (com possível risco de deglutiçäo) e lesões em tecidos moles orais decorrentes da inserção incorreta do dispositivo na boca. A correta utilização do dispositivo conforme as orientaçöes dos pesquisadores

previne que aconteça maiores problemas.

Beneficios:

Um exame clínico detalhado da boca, limpeza da superficie dental e instruçöes de higiene oral, ao término da participação no estudo.

\section{Comentários e Consideraçōes sobre a Pesquisa:}

O projeto está corretamente enquadrado na área temática e a metodologia proposta é adequada. Os antecedentes científicos justificam a execução da mesma. Possui cronograma de execuçäo detalhado, todos os documentos estão anexados, o TCLE está claro e correto. A temática proposta é original e importante para a área da saúde humana. O projeto está bem estruturado, com revisão bibliográfica adequada, pertinente ao tema e atualizada. Os pesquisadores apresentam formaçäo acadèmica adequada para desenvolver o projeto.

\section{Consideraçöes sobre os Termos de apresentação obrigatória:}

O projeto está apresentado de forma correta, cumpre todas as normativas estabelecidas pelo CEP da FORP/USP e CONEP. Os pesquisadores são habilitados para execuçäo do mesmo. Relevante para a área da saúde. Bem embasado cientificamente.

\section{Recomendaçöes:}

Aprovado.

Conclusöes ou Pendências e Lista de Inadequaçöes:

Projeto aprovado.

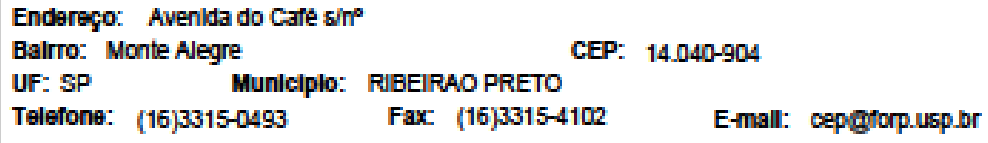




\section{USP - FACULDADE DE \\ ODONTOLOGIA DE RIBEIRÃO PRETO DA USP - FORP/USP

Continusçbo do Parecer. 2.401.193

Consideraçöes Finais a critério do CEP:

Projeto aprovado conforme deliberado na $210^{2}$ Reunião Ordinária do CEP/FORP de 27/11/2017.

Este parecer foi elaborado baseado nos documentos abaixo relacionados:

\begin{tabular}{|c|c|c|c|c|}
\hline Tipo Documento & Arquivo & Postagem & Autor & Situaçāo \\
\hline $\begin{array}{l}\text { Informações Básicas } \\
\text { do Projeto }\end{array}$ & $\begin{array}{l}\text { PB_INFORMAÇŌES_BÁSICAS_DO_P } \\
\text { ROJETO_1029491.pdf }\end{array}$ & $\begin{array}{c}13 / 11 / 2017 \\
12: 52: 09\end{array}$ & & Aceito \\
\hline $\begin{array}{l}\text { TCLE / Termos de } \\
\text { Assentimento / } \\
\text { Justificativa de } \\
\text { Ausência }\end{array}$ & TCLE_corrigido.pdf & $\begin{array}{l}13 / 11 / 2017 \\
12: 51: 13\end{array}$ & \begin{tabular}{|l} 
ANSELMO \\
AGOSTINHO \\
SIMIONATO
\end{tabular} & Aceito \\
\hline $\begin{array}{l}\text { Projeto Detalhado I } \\
\text { Brochura } \\
\text { Investigador }\end{array}$ & Projeto.pdf & $\begin{array}{l}13 / 11 / 2017 \\
09: 25: 20\end{array}$ & $\begin{array}{l}\text { Fernanda de } \\
\text { Carvalho Panzeri } \\
\text { Pires de Souza }\end{array}$ & Aceito \\
\hline Folha de Rosto & Folha_de_rosto.pdf & $\begin{array}{c}\text { 13/11/2017 } \\
09: 18: 19\end{array}$ & $\begin{array}{l}\text { Fernanda de } \\
\text { Carvalho Panzeri } \\
\text { Pires de Souza }\end{array}$ & Aceito \\
\hline
\end{tabular}

Situação do Parecer:

Aprovado

Necessita Apreciaçäo da CONEP:

Nåo

RIBEIRAO PRETO, 27 de Novembro de 2017

Assinado por:

Simone Cecilio Hallak Regalo

(Coordenador)

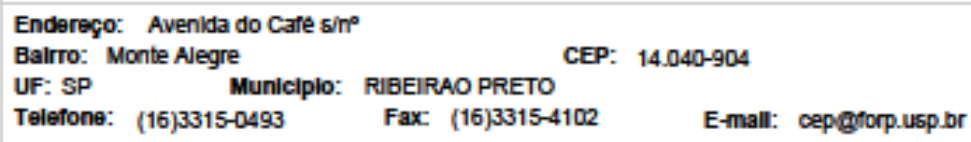


61 ANEXOS 